\title{
The Canonical Nuclear Many-Body Problem as an Effective Theory
}

\author{
W. C. Haxton and T. Luu \\ a Institute for Nuclear Theory, Box 351550, and Department of Physics, Box 351560, \\ University of Washington, Seattle, Washington 98195
}

Recently it was argued that it might be possible to treat the conventional nuclear structure problem - nonrelativistic point nucleons interacting through a static and rather singular potential - as an effective theory in a shell-model basis. In the first half of this talk we describe how such a program can be carried out for the simplest nuclei, the deuteron and ${ }^{3} \mathrm{He}$, exploiting a new numerical technique for solving the self-consistent Bloch-Horowitz equation. Some of the properties of proper effective theories are thus illustrated and contrasted with the shell model. In the second half of the talk we use these examples to return to a problem that frustrated the field three decades ago, the possibility of reducing the effective interactions problem to perturbation theory. We show, by exploiting the Talmi integral expansion, that hard-core potentials can be systematically softened by the introduction of a series of contact operators familiar from effective field theory. The coefficients of these operators can be run analytically by a renormalization group method in a scheme-independent way, with the introduction of suitable counterterms. Once these coefficients are run to the shell model scale, we show that the renormalized coefficients contain all of the information needed to evaluate perturbative insertions of the remaining soft potential. The resulting perturbative expansion is shown to converge in lowest order for the simplest nucleus, the deuteron.

\section{INTRODUCTION}

It is an honor to be able to address a distinguished audience at this celebration for Achim Richter, a very good friend and valued colleague who has done so so much to advance nuclear physics. It is also great fun to return, in this talk, to a topic that amused several of the theoreticians in this room in the 1970's, the possibility of a perturbative expansion of effective interactions and operators. The thesis of this talk is that a marriage of the formidable technology of the shell model (SM) with the modern ideas of the renormalization group and effective theory (ET) may help us make some progress on this important problem.

In the first half of this talk we contrast the conventional SM with the results of a proper ET carried out in a SM basis. We introduce a new and rather efficient numerical method for solving the self-consistent Bloch-Horowitz equation governing effective interactions and operators. The method, while numerical, systematically extracts from the excluded high-momentum space the information needed to construct effective interactions, and thus is in the spirit of ET techniques. The results for two simple nuclei, the deuteron and 
${ }^{3} \mathrm{He}$, illustrate many of the properties of correct effective theories (simple wave function evolution in the included space, nontrivial normalizations) that are absent in the shell model.

The effective interactions problem is known to be highly nonperturbative. In the second half of this talk we exploit these solutions in an effort to understand the source of the nonperturbative scattering at high momenta. The first source we encounter arises from the overbinding of harmonic oscillator wave functions. A straightforward reorganization of the Bloch-Horowitz equation - equivalent to resumming the kinetic energy operator to all orders - removes this problem. The next source is hard-core scattering. To attack this problem we build a bridge between effective field theories and potential problems by showing that the contact operators introduced in the former generate a version of the Talmi integral expansion in a SM basis. Thus a rather singular NN potential can be systematically softened by removing the lowest order Talmi integrals, replacing these with contact potentials. The effective interactions problem for these contact interactions can be solved analytically via a shell-by-shell renormalization group equation. The hard-core effective interactions problem corresponds to understanding the running of the coefficients of these contact interactions. The running can be done in a scheme-independent way - that is, the effects of the hard core on every matrix element in the SM space can be evaluated exactly - with the introduction of a finite number of higher-order contact interactions (or counterterms).

With the hard core problem thus solved, we return to the remaining soft parts of the potential, showing they can be inserted perturbatively among the infinite hard-core scattering series. The only information needed is the renormalized coefficients of the contact interactions. We illustrate, for the lowest order calculation in the deuteron, that this procedure reduces the effective interactions problem to perturbation theory.

Although the talk focuses on the simplest possible example, the techniques employed are quite general. Details not presented here can be found in [1] 3].

\section{EFFECTIVE INTERACTIONS}

Consider a collection of nonrelativistic nucleons interacting through a potential, e.g., of the Nijmegen or Argonne/Urbana type, within an infinite Hilbert space

$$
H=\frac{1}{2} \sum_{i, j=1}^{A}\left(T_{i j}+V_{i j}\right),
$$

where $T_{i j}$ is the relative nonrelativistic kinetic energy operator and $V_{i j}$ the nucleon-nucleon potential. The related SM Hamiltonian acts in a restricted space and employs a softer "effective" potential,

$$
H_{S M}=\frac{1}{2} \sum_{i, j=1}^{A}\left(T_{i j}+V_{i j}^{e f f}\right) .
$$

Motivating $H_{S M}$ is the notion that the determination of $V^{\text {eff }}$ might be simpler than solving the original A-body problem: the foundation of Brueckner theory is that highmomentum contributions to the wave function might be integrated out in a rapidly converging series in $\rho_{\text {nuclear }}$ or, equivalently, in the number of nucleons in high-momentum states interacting at one time outside the SM space. 
As will become apparent later, a typical SM space will contain explicitly $\sim 60 \%$ of the wave function that resides at long-wavelengths, thus guaranteeing at least a qualitative description of A-body correlations important to soft collective modes. The strength of the $\mathrm{SM}$ is its capacity to treat this long-wavelength part of the problem nonperturbatively: the technology for direct diagonalizations in large SM spaces is quite remarkable, including recent progress in Lanczos-based methods [ []], in treatments of light nuclei involving many shells [5], and in Monte Carlo sampling [6],7]. Unfortunately the SM - it is a model makes a number of uncontrolled approximations that distinguish it from an ET. These are discussed in Ref. [1] and include effective interactions lacking the correct functional form; a misunderstanding of the proper normalization for SM wave functions; dependence of results on fictitious parameters such as starting energies; and the almost total neglect of effective operators.

\subsection{Self-consistent Bloch-Horowitz Solutions}

The effort to reformulate the SM as an ET begins with a definition of the "SM" space. The goals of handling bound states and of generating an effective interaction that is translationally invariant leaves one sensible choice: many-body states constructed from harmonic oscillator Slater determinants. To exploit the relative/center-of-mass separability of harmonic oscillator Slater determinants, one must separate the SM and high-momentum spaces so that all configurations satisfying

$$
E \leq \Lambda_{S M} \hbar \omega_{S M}
$$

are retained in the former. For example, a SM calculation of ${ }^{16} \mathrm{O}$ with $\Lambda_{S M}=4+\Lambda_{0}$, where $\Lambda_{0}$ is the number of quanta in the ${ }^{16} \mathrm{O}$ closed shell, would include all $4 \hbar \omega_{S M}$ configurations, e.g., $0 p 0 h, 2 p 2 h$, and $4 p 4 h$ excitations of nucleons from the $1 p$ shell into the $2 s 1 d$ shell, $1 p 1 h$ excitations of a $1 s$ shell nucleon into the $3 s 2 d 1 \mathrm{~g}$ shell, etc. One can define the projection operator onto the excluded high-momentum space by

$$
Q_{S M}=Q\left(\Lambda_{S M}, b_{S M}\right) .
$$

where $b_{S M}$ is the oscillator parameter - defined here in the usual SM way for independent particle motion - and $\hbar \omega_{S M}=\frac{\hbar^{2}}{M b_{S M}^{2}}$ is the corresponding energy. Thus the included or "SM" space is defined by two parameters, $\Lambda_{S M}$ and $b_{S M}$. The preservation of translational invariance is also important numerically, as it reduces the two-body ladder to an effective one-body problem, etc.

The resulting Bloch-Horowitz $(\mathrm{BH})$ equation [8] is then

$$
\begin{aligned}
H^{e f f} & =H+H \frac{1}{E-Q_{S M} H} Q_{S M} H \\
H^{e f f}\left|\Psi_{S M}\right\rangle & =E\left|\Psi_{S M}\right\rangle \quad\left|\Psi_{S M}\right\rangle=\left(1-Q_{S M}\right)|\Psi\rangle
\end{aligned}
$$

where $|\Psi\rangle$ is the exact wave function and $H|\Psi\rangle=E|\Psi\rangle$. The difficulty posed by this equation is the appearance of the unknown energy eigenvalue in the equation for $H^{e f f}$. Thus this system must be solved self-consistently. Note that there is no explicit reference to the harmonic oscillator in this equation: it enters only implicitly through $Q_{S M}$ in distinguishing the long-wavelength "SM" space from the remainder of the Hilbert space. 
We emphasize that a proper solution of the $\mathrm{BH}$ equation must yield results (energies and operator matrix elements) that are independent of $Q_{S M}$.

A new procedure for solving this problem was introduced in Ref. [1]. It is based on a mapping of the full Hamiltonian in the high momentum space - this space can be made finite, extending to some scale $\Lambda_{\infty} \sim 3 \mathrm{GeV}$ adequate to fully resolve the hard cores of realistic potentials - into a simpler, truncated Hamiltonian via the Lanczos algorithm, followed by the demonstration that the the BH Green's function can be constructed from the Lanczos matrix as a function of $E$, thus solving the effective interactions problem. That is, one can iterate on $E$ simply by repeating a shell model calculation, as the effective interaction can be immediately generated for any desired $E$. In practice the procedure converges very rapidly.

In our view the Lanczos approach to the effective interactions problem appears to be remarkably simpler than the standard procedures of the field, particular in view of the need for extensions to multi-nucleon ladders. The traditional approach divides the effective interactions problem into an energy-independent piece (often called the Q-box) and an energy-dependent one (represented by folded diagrams). Frequently the energy dependence is removed by making a unitary transformation to a non-Hermitian effective Hamiltonian. The Lanczos procedure is also much more in the spirit of EFT: the full Hamiltonian in the high-momentum space is never constructed. Rather, it is replaced by a much smaller Lanczos matrix which contains exactly the most relevant long-wavelength information of the full matrix, the $2 n-1$ lowest moments (here $n$ represents the $n$th iteration in the Lanczos algorithm). Thus the procedure can be viewed as a numerical ET in which this information is recursively extracted. Self-consistent solutions must be obtained for each state, thus yielding a Hermitian but energy-dependent effective interaction.

\subsection{Properties of Effective Wave Functions and Operators}

A first test of the techniques outlined above is to solve the BH equation for some SMlike space to then see if the resulting self-consistent energy is, indeed, the correct value (e.g., in agreement with Faddeev or other exact calculations). For model spaces of 2, 4, 6 , and $8 \hbar \omega_{S M}$ in the case of the deuteron we obtained a binding energy of $-2.224 \mathrm{MeV}$ (using $\sqrt{2} b_{S M}=1.6 f$ and $\Lambda_{\infty}=140$ ). The exact result is $-2.2246 \mathrm{MeV}$. Our ${ }^{3} \mathrm{He}$ result for same SM spaces and $\Lambda_{\infty}=60$ is $-6.87 \mathrm{MeV}$, in agreement with the corresponding Green's function Monte Carlo (GFMC) result of $-6.87 \pm 0.03 \mathrm{MeV}$. Note that energies are variational in $\Lambda_{\infty}$, decreasing as $\Lambda_{\infty}$ is increased.

More interesting is the evolution of the wave functions, shown in Table 1, which is quite unlike that of typical shell model calculations. The wave functions obtained in different model spaces agree over overlapping parts of their Hilbert spaces. Thus as one proceeds through $2 \hbar \omega_{S M}, 4 \hbar \omega_{S M}, 6 \hbar \omega_{S M}, \ldots$ calculations, the ET wave function evolves only by adding new components in the expanded space. The normalization of the wave function grows accordingly. (This normalization must be calculated, as described below.) Thus, for ${ }^{3} \mathrm{He}$, the $0 \hbar \omega_{S M}$ ET calculation contains 0.311 of the full wave function in the effective space; the $0+2+4 \hbar \omega_{S M}$ result is 0.700 .

Although there is clearly an intimate relation between effective interactions and effective operators, it is standard in the shell model to calculate nuclear responses with bare operators, or perhaps with bare operators renormalized according to effective charges de- 
Table 1

ET results for the ${ }^{3} \mathrm{He}$ ground state wave function calculated with the Argonne $v 18$ potential. The columns on the right correspond to different choices of the ET model space, the analog of a SM space. The rows correspond to the resulting amplitudes for the designated, selected configurations. The quantities within the parentheses are the square of the norm of the effective wave function, e.g., the fraction of the ${ }^{3} \mathrm{He}$ ground state that resides in the "SM" space. The basis states are designated somewhat schematically as $|N, \alpha\rangle$, where $N$ is the total number of oscillator quanta and $\alpha$ is an index representing all other quantum numbers.

\begin{tabular}{|c|r|r|r|r|r|r|}
\hline \hline \multirow{3}{*}{ state } & \multicolumn{7}{|c|}{ amplitude } \\
\cline { 2 - 7 } & $0 \hbar \omega_{S M}$ & $2 \hbar \omega_{S M}$ & $4 \hbar \omega_{S M}$ & $6 \hbar \omega_{S M}$ & $8 \hbar \omega_{S M}$ & exact \\
\cline { 2 - 7 } & $(31.1 \%)$ & $(57.4 \%)$ & $(70.0 \%)$ & $(79.8 \%)$ & $(85.5 \%)$ & $(100 \%)$ \\
\hline$|0,1\rangle$ & 0.55791 & 0.55791 & 0.55791 & 0.55795 & 0.55791 & 0.55793 \\
\hline$|2,1\rangle$ & 0.00000 & 0.04631 & 0.04613 & 0.04618 & 0.04622 & 0.04631 \\
\hline$|2,2\rangle$ & 0.00000 & -0.48255 & -0.48237 & -0.48243 & -0.48243 & -0.48257 \\
\hline$|2,3\rangle$ & 0.00000 & 0.00729 & 0.00731 & 0.00730 & 0.00729 & 0.00729 \\
\hline$|2,4\rangle$ & 0.00000 & 0.16707 & 0.16698 & 0.16706 & 0.16706 & 0.16708 \\
\hline$|4,1\rangle$ & 0.00000 & 0.00000 & -0.02040 & -0.02042 & -0.02043 & -0.02047 \\
\hline$|4,2\rangle$ & 0.00000 & 0.00000 & 0.11267 & 0.11274 & 0.11275 & 0.11289 \\
\hline$|4,3\rangle$ & 0.00000 & 0.00000 & -0.04191 & -0.04199 & -0.04208 & -0.04228 \\
\hline$|4,4\rangle$ & 0.00000 & 0.00000 & 0.28967 & 0.28978 & 0.28978 & 0.29001 \\
\hline \hline
\end{tabular}

termined phenomenologically at $q^{2}=0$, using SM wave functions normed to 1 . As we now have a series of exact effective interactions corresponding to different model spaces, we can test the validity of this approach. The results for the elastic magnetic form factors for the deuteron and ${ }^{3} \mathrm{He}$ are shown in Fig. 1.

One sees in each case that by the time one reaches a momentum transfer $q \sim 2.5 / f$, random numbers are being generated: bare operators used in conjunction with exact effective wave functions generate results that differ by an order of magnitude, depending on the choice of the model space. This is not surprising: if one probes the nucleus at momentum transfers $\gtrsim 2 k_{F}$, where $k_{F}$ is the Fermi momentum, most of the resulting amplitude should reside outside the long-wavelength model space. That is, the strength resides entirely in the effective contributions to the operator. If these components are ignored, the results have to be in error.

Clearly the effective interaction and effective operator have to be treated consistently and on the same footing. If $\hat{O}$ is the bare operator, one finds

$$
\left\langle\Psi_{f}|\hat{O}| \Psi_{i}\right\rangle \equiv\left\langle\Psi_{f}^{e f f}\left|\hat{O}^{e f f}\right| \Psi_{i}^{e f f}\right\rangle
$$

where

$$
\hat{O}^{e f f}=\left(1+H Q_{S M} \frac{1}{E_{f}-H Q_{S M}}\right) \hat{O}\left(1+\frac{1}{E_{i}-Q_{S M} H} Q_{S M} H\right)
$$


and where the effective wave function normalization of $\left|\Psi_{i}^{e f f}\right\rangle$ and $\left|\Psi_{f}^{e f f}\right\rangle$, mentioned earlier, must be determined using the effective operator $\hat{1}$, e.g.,

$$
1=\left\langle\Psi_{i} \mid \Psi_{i}\right\rangle=\left\langle\Psi_{i}^{e f f}\left|\left(1+H Q_{S M} \frac{1}{E_{i}-H Q_{S M}}\right)\left(1+\frac{1}{E_{i}-Q_{S M} H} Q_{S M} H\right)\right| \Psi_{i}^{e f f}\right\rangle
$$

These expressions can be evaluated with the Lanczos Green's function methods described earlier. When this is done, all of the effective calculations, regardless of the choice of the model space, yield the same result, given by the solid lines in Fig. 1.

We would argue, based on this example, that many persistent problems in nuclear physics - ranging from the renormalization of $g_{A}$ in $\beta$ decay to the systematic differences between measured and calculated M1 electromagnetic form factors — very likely are due to naive treatments of operators, treatments that fail to satisfy the basic rules of ETs. It should be apparent from the above example that no amount of work on $H^{\text {eff }}$ will help with this problem. What is necessary is a diagrammatic basis for generating $H^{e f f}$ that can be applied in exactly the same way to evolving $\hat{O}^{e f f}$. From this perspective, phenomenological derivations of $H^{e f f}$ by fitting binding energies and other static properties of nuclei are not terribly helpful, unless one intends to simultaneously find phenomenological renormalizations for each desired operator in each $q^{2}$ range of interest.

The point of this discussion has been largely pedagogical. To our knowledge the examples given above are the only ones in the classical nuclear physics literature in which a "model space" calculation has been formulated in a way that satisfies the basic rules of ET. The results - particularly the invariance of energies and operator matrix elements under changes in how we define $Q$ - are obvious from the perspective of ET. The fact that standard techniques like the SM violate so many of the rules of ETs is cause for optimism: much can be done to improve the rigor of such nuclear physics tools. As we have demonstrated here, new numerical methods can be developed to handle the full ET problem at the cost of only modest additional effort.

\section{RESUMMATIONS: LONG AND SHORT DISTANCES}

The effort to derive SM effective interactions directly from realistic NN interactions was largely abandoned in the early 1970's when it became apparent that the high-momentum scattering problem was nonperturbative. A series of efforts involving perturbative expansions in either the potential $V$ or the corresponding $G$-matrix (the sum of the two-body ladder diagrams) yielded discouraging results, with higher-order terms often dominating over lower-order ones 10]. In particular Shucan and Weidenmuller showed that any overlap in the spectra of included and excluded, high-momentum states would lead to a nonperturbative series [11, 12]. Thus, the uncontrolled approximations in the SM approach grew out of necessity: it became a largely phenomenological tool in part because early efforts to provide a more rigorous basis for the model ended in failure.

Given our two SM-like ET calculations, the deuteron and ${ }^{3} \mathrm{He}$, it is interesting to return to this old problem of the nonperturbative behavior of the nonrelativistic manybody problem, using these examples as laboratories. Today's challenge, in fact, is more modest than that of the 1970's: modern computers coupled with new algorithms, like the Lanczos solution of the $\mathrm{BH}$ equation introduced above, allow us to solve rather complex 


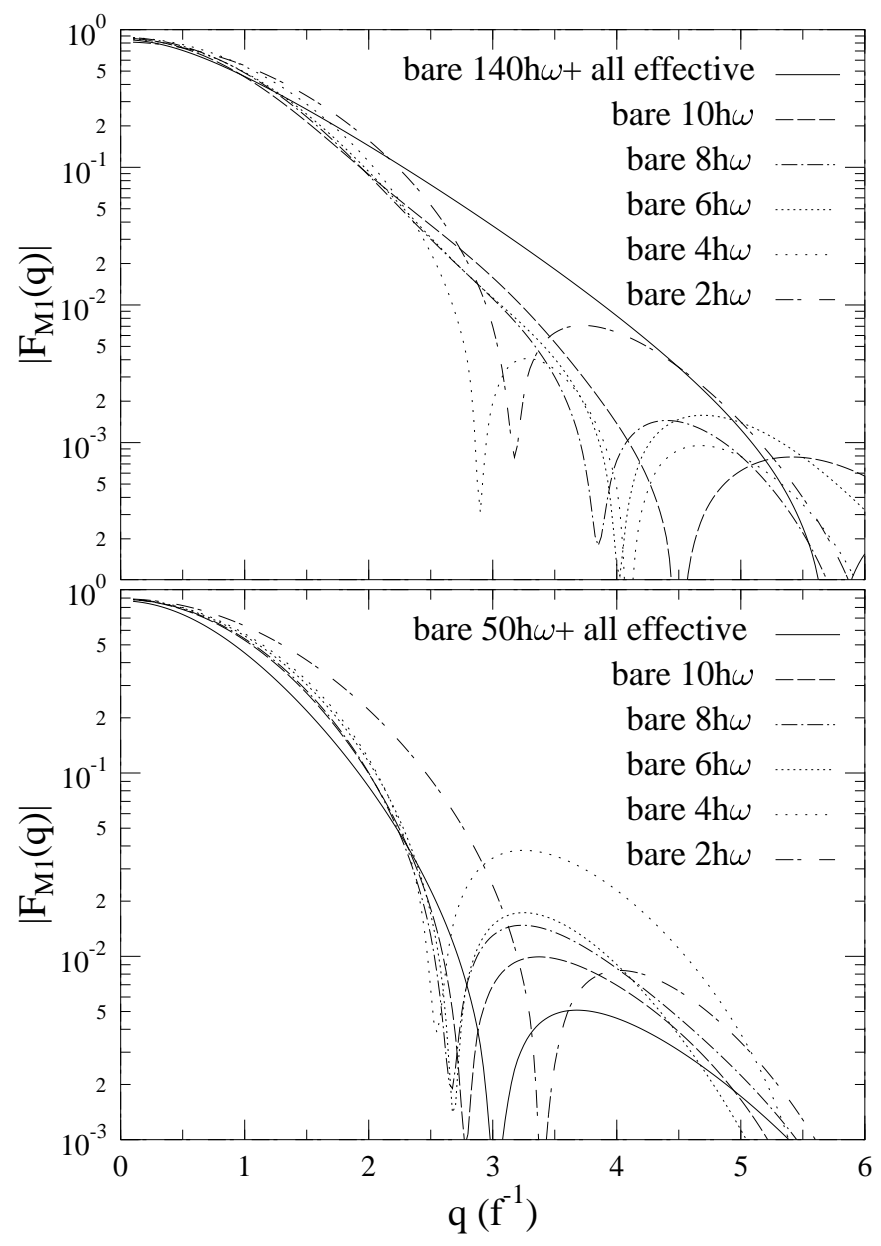

Figure 1. The magnetic elastic form factor for the deuteron (top) and ${ }^{3} \mathrm{He}$ (bottom) calculated with the exact $H^{e f f}$, SM wave functions normalized to unity, and a bare operator are compared to the exact result (solid line) [9, [1]. When effective operators and the proper wave function normalizations are used, all results become identical to the solid line.

nonperturbative problems. To extend the calculations above to ${ }^{4} \mathrm{He}$ and heavier nuclei one has to achieve only modest increases in efficiency, such as a substantial lowering of $\Lambda_{\infty}$. Below we summarize some of our most recent work [3] that suggests that the goals of the 1970's - a perturbative treatment of the nonrelativistic nuclear physics problem - may not be out of the question. Through renormalization group and other techniques, the most severely nonperturbative contributions to the potential can be systematically extracted and treated. In effect, we try to show how many of the ideas of EFT can be adapted to the nuclear potential problem, without abandoning the basic idea of the SM that rigorous nonperturbative diagonalizations in a long-wavelength included space are important to the physics. 


\subsection{Harmonic Oscillator Overbinding and Kinetic Energy Resummations}

We begin by studying the behavior of SM effective interaction matrix elements $\left\langle\alpha\left|H^{e f f}\right| \beta\right\rangle$ in a perturbative expansion of the $\mathrm{BH}$ propagator

$$
\frac{1}{E-Q H}=\frac{1}{E-H_{0}}+\frac{1}{E-H_{0}} Q\left(V-V_{0}\right) \frac{1}{E-H_{0}}+\cdots,
$$

evaluating the series term by term. Here $H_{0}$ and $V_{0}$ are the harmonic oscillator Hamiltonian and potential. We thus lower $\Lambda_{\infty}$ and test whether states above $\Lambda_{\infty}$ can be treated perturbatively.

Once a scale of $\Lambda_{\infty} \sim 70$ is reached, striking differences appear in the rate of convergence of different matrix elements. All are greatly improved in first and second order perturbation theory. Those matrix elements well below the "boundary" at $\Lambda_{\infty}$ converge quickly to their correct values in this way. However, those matrix elements $\left\langle\alpha\left|H^{e f f}\right| \beta\right\rangle$ where either $|\alpha\rangle$ or $|\beta\rangle$ resides in the last shell (with $N=\Lambda_{\infty}$ ) converge only very slowly to the correct values, after the initial improvement in low-order perturbation theory. Typically $\sim 10^{3}$ orders of perturbation theory are required to produce the correct value. This clearly suggests that slow convergence is associated with the relative kinetic energy operator $Q T$ contribution to $Q H$, as the only transitions to states outside the Hilbert space generated by this operator have $\Delta n=1$. At large $r$ the strength of this transition becomes quite large, $-Q V_{0}(r)|\alpha\rangle$, reflecting the unphysical asymptotic behavior of harmonic oscillator wave functions. This amplitude propagates nonperturbatively as $\left(V-V_{0}\right) / H_{0} \sim 1$. Eventually enough high-momentum harmonic oscillator wave functions are coupled together to produce the softer asymptotic fall-off characteristic of the correct bound-state wave function.

As discussed in Ref. [3], the required resummation is guided by the observation that the true potential, $V(r)$, falls off properly at large $r$. Thus a reorganization of the $\mathrm{BH}$ equation in which the propagator is always sandwiched between $V(r)$ should remove the unwanted propagation. This leads to the following recasting of the $\mathrm{BH}$ equation:

$$
\begin{aligned}
\left\langle\alpha\left|H^{e f f}\right| \beta\right\rangle= & \langle\alpha|T| \beta\rangle+(\langle\hat{\alpha}|-\langle\alpha|) E-T(|\hat{\beta}\rangle-|\beta\rangle) \\
& +\left\langle\hat{\alpha}\left|V+V \frac{1}{E-Q H} Q V\right| \hat{\beta}\right\rangle
\end{aligned}
$$

where

$$
|\hat{\alpha}\rangle=\frac{E}{E-Q T}|\alpha\rangle
$$

If $\alpha$ and $\beta$ are not in the last shell, $|\hat{\alpha}\rangle=|\alpha\rangle$ and $|\hat{\beta}\rangle=|\beta\rangle$, so that the above rewriting of the $\mathrm{BH}$ equation reduces to the original form. Otherwise, a modified wave function generated by Eq. (11) must be used. This rather simple resummation removes the worst though not the most interesting - source of nonperturbative behavior in the BH equation.

It should be clear that a large reduction in the scale $\Lambda_{\infty}$ at the cost of an evaluation of Eq. (I1) represents a tremendous savings: in place of a dense matrix $Q H$ whose elements have to be evaluated numerically, we have a sparse matrix $Q T$ whose matrix elements are known analytically. For example, in the case of the deuteron, $Q T$ is triadiagonal in the harmonic oscillator basis, allowing us to write the Green's function in a continued fraction expansion. 


\subsection{Talmi Integrals, Renormalization Group, and the Hard Core}

Once the replacement $|\alpha\rangle \rightarrow|\hat{\alpha}\rangle$ is made, $\Lambda_{\infty}$ can be lowered to $\sim 40$ while maintaining $1 \mathrm{keV}$ accuracy, if one works to third order in perturbation theory. But with further lowering, errors arise in the perturbative expansion that persist even if calculations are carried to very high order. At $\Lambda_{\infty}=30$, third order perturbation theory reduces $\sim$ $10 \%$ errors in $\left\langle n^{\prime}=1 l^{\prime}=0\left|H^{e f f}\right| n=1 l=0\right\rangle$ to $\sim 0.2 \%$. But an error in excess of $0.1 \%$ - corresponding to $50 \mathrm{keV}$ - persists after 10 additional orders of perturbation. Numerically one can verify that the nonperturbative tail is generated by the scattering at very small $r$. As expected, the nonperturbative contributions to $s-d$ matrix elements are much smaller than those for $\left\langle n^{\prime}=1 l^{\prime}=0\left|H^{e f f}\right| n=1 l=0\right\rangle$ and other $s-s$ matrix elements.

We think this hard-core problem can be solved. To keep the presentation reasonable, below we concentrate primarily on the lowest order (LO) treatment. (The NLO and NNLO results are given in Ref. [3].) One begins with the Talmi integral expansion for radial harmonic oscillator matrix elements in the relative coordinate

$$
\begin{aligned}
\left\langle n^{\prime} l^{\prime}\left|V_{s r}(r)\right| n l\right\rangle & =\int_{0}^{\infty} R_{n^{\prime} l^{\prime}}(r) V_{s r}(r) R_{n l}(r) r^{2} d r \\
& =\sum_{p} B\left(n^{\prime}, l^{\prime} ; n, l ; p\right) I_{p}(b)
\end{aligned}
$$

where

$$
\begin{aligned}
I_{p}(b) & =\frac{2}{\Gamma(p+3 / 2)} \int_{0}^{\infty} e^{-r^{2} / b^{2}} V_{s r}(r) \frac{r^{2 p+2} d r}{b^{2 p+3}}, \\
b & =\sqrt{2} b_{S M} .
\end{aligned}
$$

The $B\left(n^{\prime}, l^{\prime} ; n, l ; p\right)$ are known analytically $[13]$. Here $V_{s r}(r)$ denotes the short-range contributions to the potential (we return to this point below). The integrals $I_{p}$ can be viewed as a systematic expansion of the nonperturbative hard core in terms of the parameter $\left(r_{c} / b\right)^{2}$, where $r_{c}$ is a distance associated with the size of the hard core that remains unresolved at scale $\Lambda_{\infty}$. Thus the LO term in the expansion is

$$
I_{p=0}^{(0)}=\frac{2}{\Gamma(3 / 2)} \frac{1}{b^{3}} \int_{0}^{\infty} V_{s r}(r) r^{2} d r
$$

while in NLO one includes

$$
\begin{aligned}
& I_{p=0}^{(1)}=\frac{2}{\Gamma(3 / 2)} \frac{1}{b^{3}} \int_{0}^{\infty} V_{s r}(r) r^{2}\left(1-\frac{r^{2}}{b^{2}}\right) d r \\
& I_{p=1}^{(0)}=\frac{2}{\Gamma(5 / 2)} \frac{1}{b^{5}} \int_{0}^{\infty} V_{s r}(r) r^{4} d r .
\end{aligned}
$$

In NNLO one obtains

$$
\begin{aligned}
& I_{p=0}^{(2)}=\frac{2}{\Gamma(3 / 2)} \frac{1}{b^{3}} \int_{0}^{\infty} V_{s r}(r) r^{2}\left(1-\frac{r^{2}}{b^{2}}+\frac{r^{4}}{2 b^{4}}\right) d r \\
& I_{p=1}^{(1)}=\frac{2}{\Gamma(5 / 2)} \frac{1}{b^{5}} \int_{0}^{\infty} V_{s r}(r) r^{4}\left(1-\frac{r^{2}}{b^{2}}\right) d r \\
& I_{p=2}^{(0)}=\frac{2}{\Gamma(7 / 2)} \frac{1}{b^{7}} \int_{0}^{\infty} V_{s r}(r) r^{6} d r .
\end{aligned}
$$


and so on.

Now the radial component of the $s$-wave potential $V_{0} \delta(\mathbf{r})$ is

$$
V_{\delta}^{(0)}(r)=V_{0} \frac{1}{4 \pi r^{2}} \delta(r) .
$$

Substituting this into Eq. (12) yields

$$
\left\langle n^{\prime} l^{\prime}=0\left|V_{\delta}^{(0)}(r)\right| n l=0\right\rangle=B\left(n^{\prime}, 0 ; n, 0 ; 0\right) I_{0}^{\delta}(b)
$$

where

$$
I_{0}^{\delta}(b)=\frac{2}{\Gamma(3 / 2)} \frac{1}{4 \pi b^{3}} V_{0}
$$

It follows that the contact interaction will produce the exact lowest-order Talmi integral contribution (Eq. (14)) to $V_{s r}$ provided

$$
V_{0}=4 \pi \int_{0}^{\infty} V_{s r}(r) r^{2} d r .
$$

Using a normalization that will be convenient later, we can then rewrite $V_{s r}(\mathbf{r})$ as

$$
V_{s r}(\mathbf{r})=V_{s r}^{(1)}(\mathbf{r})+b^{3} \frac{\pi^{2}}{2} \hbar \omega a_{0}^{s s}\left(\Lambda_{\infty}\right) \delta(\mathbf{r})
$$

where $\omega=\frac{\omega_{S M}}{2}$ and $V_{s r}^{(1)}$ is a new potential whose leading-order behavior is of order $\left(r_{c} / b\right)^{2}$ relative to $V_{s r}$ (determined by subtracting from the original Talmi integral expression for $V_{s r}$ the contribution from Eq. (14)). The coefficient $a_{0}^{s s}\left(\Lambda_{\infty}\right)$ is a dimensionless coupling. (Note that throughout this discussion, we have suppressed the spin and isospin of $V_{s r}(\mathbf{r})$. Thus $V_{s r}(r)$ represents the radial function obtained after spin and isospin matrix elements have been taken. For example, the components of the av18 potential contributing to $s-s$ transitions is

$$
V_{s r}(\mathbf{r})=V_{1}(r)+V_{2}(r) \vec{\tau}_{1} \cdot \vec{\tau}_{2}+V_{3}(r) \vec{\sigma}_{1} \cdot \vec{\sigma}_{2}+V_{4}(r) \vec{\sigma}_{1} \cdot \vec{\sigma}_{2} \vec{\tau}_{1} \cdot \vec{\tau}_{2}
$$

so that

$$
V_{s r}(r)=V_{1}(r)-3 V_{2}(r)+V_{3}(r)-3 V_{4}(r)
$$

for the deuteron $(l=0, s=1, t=0)$. $)$

The above procedure is, in fact, general. The NLO contribution (Eq. (15)) can be removed from $s-s$ wave deuteron matrix elements of $V_{s r}(\mathbf{r})$ by introducing a second, higher-order contact operator

$$
a_{2}^{s s}\left(\Lambda_{\infty}\right) \frac{1}{2}\left(\overleftarrow{\nabla}^{2} \delta(\mathbf{r})+\delta(\mathbf{r}) \vec{\nabla}^{2}\right)
$$

where

$$
a_{2}^{s s}\left(\Lambda_{\infty}\right)=\frac{8}{3 \pi b^{5} \hbar \omega} \int_{0}^{\infty} V_{s r}(r) r^{4} d r
$$


while the NNLO contributions can be removed by

$$
a_{4}^{s S}\left(\Lambda_{\infty}\right)\left(\overleftarrow{\nabla}^{2} \delta(\mathbf{r}) \vec{\nabla}^{2}+\frac{3}{10}\left(\overleftarrow{\nabla}^{4} \delta(\mathbf{r})+\delta(\mathbf{r}) \vec{\nabla}^{4}\right)\right)
$$

where

$$
a_{4}^{s s}\left(\Lambda_{\infty}\right)=\frac{2}{9 \pi b^{7} \hbar \omega} \int_{0}^{\infty} V_{s r}(r) r^{6} d r .
$$

Likewise there are coeficients of additional contact operators (denoted $a_{2}^{s d}, a_{4}^{s d}$, and $a_{4}^{d d}$ in Ref. [3]) that remove the Talmi integral contributions to $s-d$ and $d-d$ matrix elements to NNLO.

Up to this point we have simply rewritten the "bare" potential - the potential that acts in the space defined by $\Lambda_{\infty}$ - in an entirely equivalent form, exploiting the Talmi integral expansion and the fact that the unique translationally-invariant two-body contact operators generate those Talmi integrals. Now we consider, in LO, the effects on $a_{0}^{s s}$ of integrating out the single shell at $\Lambda_{\infty}$, thereby mapping the original problem into an effective one with $\Lambda_{\infty} \rightarrow \Lambda_{\infty}-2$. The resulting $\mathrm{BH}$ equation instructs one to simply renormalize the coefficient $a_{0}^{s s}(\Lambda)$ in LO [3],

$$
a_{0}^{s s}(\Lambda-2)=a_{0}^{s s}(\Lambda)+\frac{\Gamma\left(\frac{\Lambda+3}{2}\right)}{\left(\frac{\Lambda}{2}\right) !} \frac{a_{0}^{s s}(\Lambda)^{2}}{E_{0}-\left(\Lambda+\frac{3}{2}\right)-a_{0}^{s s}(\Lambda) \frac{\Gamma \frac{\left(\frac{\Lambda+3}{2}\right)}{\left(\frac{\Lambda}{2}\right) !}}{10}}
$$

where $E_{0}=E / \hbar \omega$ is a dimensionless energy. ( $E$ is the BH energy being determined selfconsistently.) This leading-order RG equation is an operator equation, properly correcting every matrix element in the new space below $\Lambda_{\infty}-2$. Clearly this difference equation can be used to run $a_{0}^{s s}\left(\Lambda_{\infty}\right)$ to any desired new scale, including $\Lambda_{S M}$.

While the RG equation is a difference equation, the connections with EFT become even clearer by introducing the natural definition of the derivative

$$
\frac{d a_{0}^{s s}(\Lambda)}{d \Lambda} \equiv \frac{a_{0}^{s s}(\Lambda)-a_{0}^{s s}(\Lambda-2)}{2} .
$$

As will be apparent from results given below, the terms in the denominator of Eq. (28) that compete with $\Lambda$ are one power lower in $\Lambda$. Thus for large $\Lambda$ the RG equation can be written in a more conventional form

$$
\Lambda \frac{d a_{0}^{s s}(\Lambda)}{d \Lambda} \sim \frac{1}{2} \sqrt{\frac{\Lambda}{2}} a_{0}^{s s}(\Lambda)^{2}
$$

which has the solution

$$
a_{0}^{s s}(\Lambda) \sim-\sqrt{\frac{2}{\Lambda}} .
$$

(The RG equation can also be integrated exactly, yielding a slightly more complicated form.) As $\Lambda$ is a dimensionless nonrelativistic energy, this is equivalent to running as $1 / p$, where $p$ is the momentum, a result familiar from EFT. Similarly, $a_{2}^{s s}$ runs as $\Lambda^{-3 / 2}$ and $a_{4}^{s s}$ as $\Lambda^{-5 / 2}$. 
This procedure is followed through order $(r / b)^{4}$ in Ref. [3], which involves a series of coupled RG equations for $a_{0}^{s s}, a_{2}^{s s}, a_{4}^{s s}, a_{2}^{s d}, a_{4}^{s d}$, and $a_{4}^{d d}$. Beyond lowest order the running is no longer automatically scheme independent, but independence can be restored by introducing a finite number of higher derivative counterterms in NLO and NNLO. We also stress that the analogy between EFT approaches, where the coefficients of contact terms are determined by fitting data, and the Talmi integral expansion is very close. In NNLO, for example, the three lowest Talmi integrals completely determine the set of matrix elements coupling the $n=1$ and $n=2$ oscillator shells. We can fit our ss bare contact potential to those matrix elements, then use the RG equation to run this potential to the SM scale, arriving at the same point as our EFT colleagues. It is thus apparent that the detailed short-range form of the bare potential is irrelevant to shell-model-inspired effective theories: the needed quantities are Talmi integrals over the short-range part of the potential. Any change in the detailed radial behavior of the potential that preserves the value of the Talmi integrals will also leave the low-energy theory unchanged.

\subsection{Perturbation Theory}

In the above discussion we were careful to carry through the subscript $s r$ on our Talmi integrals, denoting the fact that, at the outset, we divide the potential in some smooth way into a long-range piece and into a short-range component. It is clearly the short-range component - that rather singular part of the bare potential that is unresolved at the scale $\Lambda_{S M}$ - that should be represented by the contact operator expansion. And clearly, while we have just solved the short-range effective interactions problem exactly, we are left with a gentler long-range potential

$$
V_{l r}\left(\Lambda_{\infty}\right)=V-V_{s r}\left(\Lambda_{\infty}\right)
$$

whose effects have not been evaluated. This potential, if we have defined $s r$ properly, is a soft potential that can be handled very well within the SM space: this is where we exploit the marvelous technology of the SM. But left over is a small contribution that couples the SM and high momentum spaces. How is this treated?

The procedure is an expansion of the $\mathrm{BH}$ propagator

$$
Q H=Q\left(H_{0}+V_{\delta}\right)+Q\left(V_{l r}-V_{0}\right)
$$

treating the second term perturbatively. This corresponds to insertions of the soft potential into an infinite series of hard-core scatterings. Thus the lowest order term is

$$
V_{\delta}^{e f f}=V_{\delta}+V_{\delta} \frac{1}{E-\left(H_{0}+Q V_{\delta}\right)} Q V_{\delta}
$$

the solution of which is just our renormalized contact interaction. The higher-order terms could be easily evaluated if we had a convenient expression for the Green's function appearing above. Such an expression exists and depends quite simply on the renormalized contact interaction coefficients. The expressions can be found in Ref. [3].

The net result for the deuteron ground state energy is shown in Fig. 2. This calculation employs a $10 \hbar \omega \mathrm{SM}$ space. If one were to try to evaluate the SM effective interaction perturbatively, the solid line is obtained. The answer oscillates rather wildly, order by order in perturbation theory, failing to converge even after 20 orders of perturbation 
theory. In contrast, the dashed line is the result of the procedure described here. The oscillations damp quickly to an answer than is accurate to a few tens of kilovolts. It appears, even in lowest order, that rather accurate results can be obtained perturbatively.

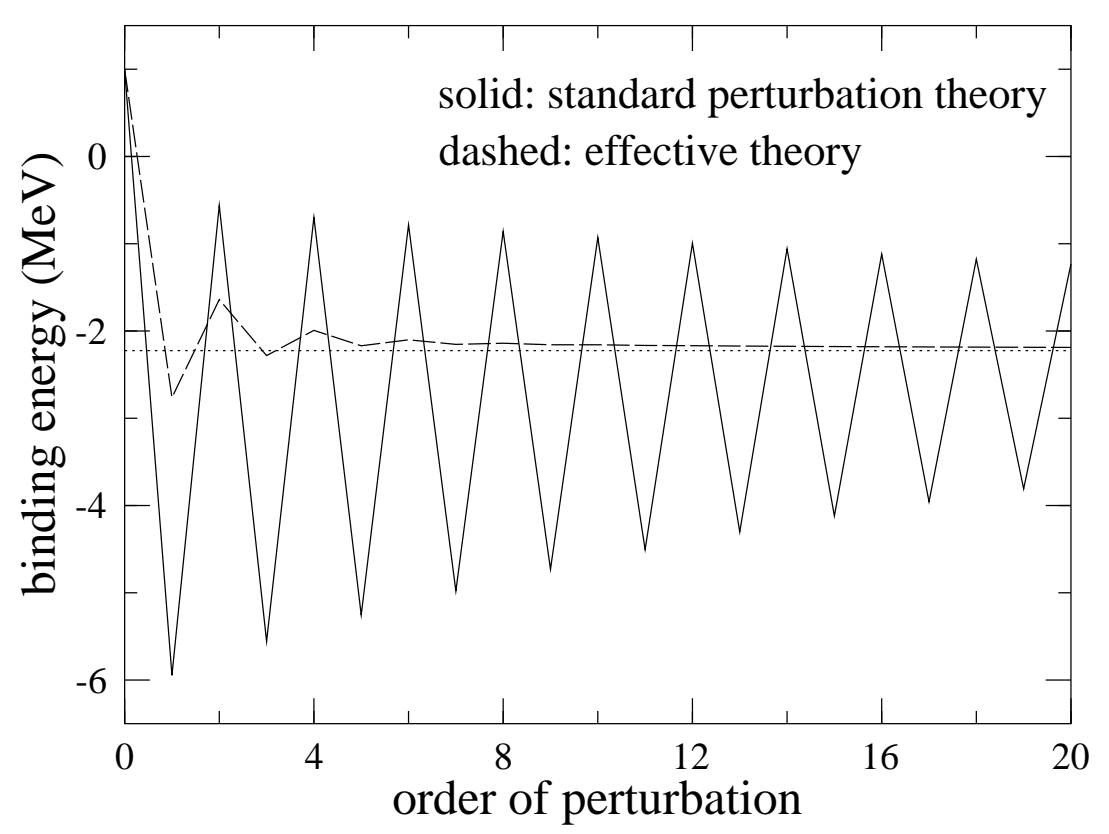

Figure 2. Calculations of the binding energy of deuterium in a $10 \hbar \omega_{S M}$ included space augmented by perturbation theory. The solid result is a standard perturbation treatment around the harmonic oscillator potential. The answer is very slowly converging. The dashed line is the ET result in which the hard core (defined in this application as the potential within $0.7 \mathrm{f}$ ) is first treated via the LO renormalization group procedure discussed in the text: the hard core is summed to all orders analytically, and then the effects of the remaining softer potential are evaluated perturbatively. The convergence improves remarkably. The corresponding exact result $\left(\Lambda_{\infty}=200\right)$ is shown by the dotted line.

\section{OUTLOOK}

The extensions to three- and four-body clusters have not yet been carried out, though nothing in the procedures is specific to the deuteron. The RG equations will, of course, involve the full set of general three- and four-body translationally invariant contact operators that can be constructed, just as EFT treatments of the three-body problem were required to introduce three-body contact terms. Thus the RG equations become more complicated, but not conceptually more difficult. If one began with a bare interaction that includes a short-ranged three-body force, the coefficients of the three-body contact terms would initially be nonzero, then evolve. If there is no such force, the initial value is zero, so that the three-body effective interaction is then entirely induced. 
If this program can be extended to heavier systems, some remarkable simplifications might occur. There exists no LO interaction that involves more than four nucleons: at most four nucleons can exist in a relative $s$-state. Thus, the LO hard-core scattering problem in $\mathrm{Pb}$ is no more difficult than the corresponding problem in ${ }^{4} \mathrm{He}$, in some sense. This is an explicit realization of Brueckner's idea of a cluster expansion at high momenta. If such a system could be reduced to perturbation theory, as we have achieved in the deuteron, some apparently unsolvable problems (such as how to properly handle the projection operator $Q$ in such systems) might be overcome. Clearly there are many hurdles before one gets to this point, but we are cautiously optimistic that more progress will be made.

In closing, it has been a great pleasure to speak at this meeting is honor of a distinguished colleague and friend. We thank Achim Richter and the organizers for the opportunity to discuss this work.

\section{ACKNOWLEDGEMENTS}

This work was supported in part by the US Department of Energy and by the University of Washington through its Royalty Research Foundation. We thank our effective field theory colleagues, particularly Martin Savage, for helpful discussions.

\section{REFERENCES}

1. W. C. Haxton and C. L. Song, Phys. Rev. Lett. 84 (2000) 5454.

2. S. R. Beane, P. F. Bedaque, W. C. Haxton, D. R. Phillips, and M. J. Savage, to be published in the Boris Ioffe Festschrift, ed. M. Shifman (World Scientific, Singapore, 2001).

3. W. C. Haxton and T. Luu, in preparation.

4. E. Caurier et al., Phys. Rev. C59 (1999) 2033.

5. P. Navratil and B. R. Barrett, Phys. Rev. C59 (1999) 1906.

6. S. E. Koonin, D. J. Dean, and K. Langanke, Phys. Rept. 278 (1997) 1.

7. M. Honma, T. Mizusaki, and T. Otsuka, Phys. Rev. Lett. 77 (1996) 3315.

8. C. Bloch and J. Horowitz, Nucl. Phys. 8 (1958) 91.

9. W. C. Haxton and C. L. Song, in Nuclear Physics with Effective Field Theory, ed. P. Bedaque et al. (World Scientific, Singapore, 2000) p. 317.

10. B. R. Barrett, Czech. J. of Phys. 49 (1999) 1.

11. T. H. Shucan and H. A. Weidenmuller, Ann. Phys. (NY) 73 (1972) 108.

12. T. H. Shucan and H. A. Weidenmuller, Ann. Phys. (NY) 76 (1973) 483.

13. T. A. Brody and M. Moshinsky, Tables of Oscillator Brackets for Nuclear Shell-Model Calculations, Gordon and Breach, New York, 1967. 